\title{
Surface Crystallography by X-ray Diffraction
}

\section{François Grey and Robert Feidenhans'I, Roskilde}

\author{
(Risø National Laboratory)
}

It has been said that all science is either physics or stamp collecting. If so, then surface science has consisted mainly of stamp collecting for the last 20 years. The collection is a vast and complex one, because crystal surfaces display a bewildering variety of structures which depend delicately on, for example, the crystal temperature or the amount of any adsorbed atoms on the surface. To extract some physics from this collection, scientists require reliable models of the surface atomic geometry. Such models have proved difficult to obtain experimentally.

The ideal surface of a crystal - the surface obtained by simply terminating the bulk crystal structure at a particular crystallographic plane - is rarely an energetically favourable arrangement for the surface atoms. To achieve a more stable configuration, the surface will often undergo a reconstruction, which can involve quite dramatic changes of the surface atomic geometry. A classical example, which illustrates the task facing the surface crystallographer, is the $7 \times 7$ reconstruction of the (111) crystallographic surface of silicon. This surface can be produced by cleaving $\mathrm{Si}$ in ultra-high vacuum and heating the crystal briefly to $400^{\circ} \mathrm{C}$. The notation $7 \times 7$ refers to the size of the reconstructed unit cell relative to the $1 \times 1$ unit cell of the ideal surface. Assuming that the reconstruction affects the first few layers of the crystal, the $7 \times 7$ unit cell contains well over 100 atoms. Such unit cells are more reminiscent of crystals of large organic molecules than of simple elements.

Given the long history of X-ray diffraction, and its prime role in the determination of complex structures such as proteins, the technique would seem an obvious candidate for surface structure determination. It is therefore somewhat surprising to find that the first attempt by Marra and Eisenberger [1] to solve a surface structure by X-ray diffraction dates from as recently as 1981. Part of the explanation for the tardy development of this technique is that the dif-

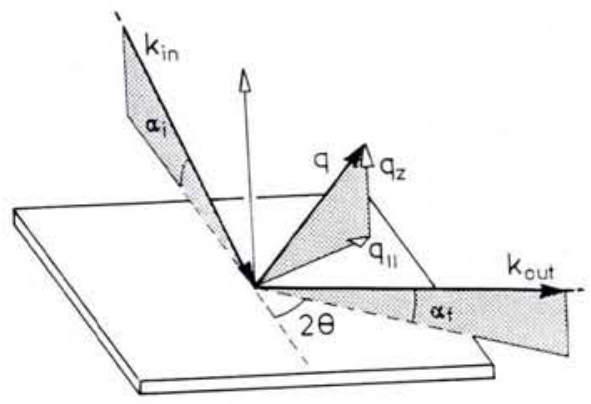

Fig. 1 - The diffraction geometry. The monochromatic $X$-ray beam is incident at an angle $\alpha_{i}$ to the surface. After being diffracted through an angle $2 \theta$ by the reconstructed surface, the $X$-rays are detected at an exit angle $\alpha_{f}$ to the surface. $\alpha_{i}$ and $\alpha_{f}$ are typically of the order $0.5^{\circ}$. The in-plane component, $\boldsymbol{q}_{1}$, of the scattering vector $\boldsymbol{q}$ is a reciprocal lattice vector of the surface lattice; the component $\boldsymbol{q}_{2}$ perpendicular to the surface can be varied by altering $\alpha_{i}$ or $\alpha_{f}$, in order to probe along a rod in reciprocal space.

fracted X-ray signal from a single atomic layer is very weak. If the first layer of atoms from a $1 \mathrm{~mm}^{2}$ region of the surface were collected into a ball it would measure only a few microns in diameter; this effective crystal size is the lower limit of what can be studied with conventional X-ray sources. That is why, to date, most surface X-ray diffraction studies have been made at synchrotron $\mathrm{X}$-ray facilities.

Intense X-ray sources though, are not the only requirement for surface crystallographic studies. The technique must also be made surface sensitive. This is because $\mathrm{X}$-rays probe tens of microns into the bulk of the crystal. Reconstructed surfaces provide a unique means of distinguishing the scattering from the surface from the much more intense bulk scattering. The larger periodicity of the reconstructed surface layer leads to extra Bragg reflections, well separated from the bulk peaks. In terms of the crystallographic Miller indices $h, k$ of the ideal surface, these extra reflections have fractional indices, and are referred to as fractional-order reflections. (For the non-specialist, the con- ventional Miller indices $h k \ell$ of a crystal plane can most easily be understood as the shortest reciprocal lattice vector normal to it.)

Without further precaution, however, the diffuse $X$-ray scattering from the bulk will swamp all but the most intense fractional-order reflections. The solution to this problem is to perform the diffraction experiment under grazing angles of incidence, thus limiting the $\mathrm{X}$-ray penetration depth considerably. The diffraction geometry is shown in Fig. 1. In particular, below a certain critical angle of incidence, typically a fraction of a degree, the $\mathrm{X}$-rays are totally reflected from the surface. This phenomenon is the direct analogue of total internal reflection in optics, but occurs externally because the refractive index of materials for X-rays is slightly less than one:

$n=1-\delta, \delta=4.49 \times 10^{-6} \mathrm{NZ}^{2}$

$N$ : number of atoms per $\AA^{3}$

$Z$ : number of electrons per atom

$\lambda$ : wavelength in $\AA$

so that, in the small angle approximation, the critical angle is:

$$
\alpha_{\mathrm{c}}=\sqrt{ } 2 \delta \text {, }
$$

typically of the order of $0.5^{\circ}$.

Under the conditions of total external reflection, the refracted wave is inhomogeneous, and exponentially damped in the direction perpendicular to the surface. The penetration depth is then typically only a few tens of Angstroms. Although this grazing incidence geometry is not essential for observing a surface-diffracted signal, it has proved to be important for obtaining large crystallographic data sets with sufficient accuracy to perform detailed structural analysis.

The weakness of the diffracted X-ray signal from a surface has one very positive aspect: the intensity is so low that multiple scattering can safely be neglected, and so analysis based on the Born approximation is applicable. The simplicity of the underlying scattering theory is the crux of the method: it is possible to analyse the results of surface $X$-ray diffraction experiments in great detail, with very modest computational means. 
This contrasts sharply with the standard technique of surface crystallography, low energy electron diffraction (LEED). Low energy electrons are a surface sensitive probe because of their strong interaction with matter, but this strong interaction leads to multiple diffraction and refraction effects. Although LEED can reveal the symmetry of the surface structure, giving the size and orientation of the surface unit cell, it is difficult to deduce reliable models for the atomic geometry from such measurements.

On the other hand, the apparatus for surface X-ray diffraction is considerably more complex and costly than the standard LEED equipment. The experiment must combine the full freedom and accuracy of an X-ray diffractometer with the ultra-high vacuum technology necessary for keeping the surfaces clean. The simplest solution to these technical

Fig. 2 - The Patterson function of Ge(001)$2 \times 1$ and dimer model. The Patterson $P(r)$ is the Fourier transform of the measured structure factor intensities:

$$
P(r) \sim \sum_{h k} / F_{h k} /\left.\right|^{2} \cos [2 \pi(h x+k y)]
$$

Positive peaks correspond to interatomic vectors in the unit cell. Because integerorder reflections are not included, only layers significantly different from the bulk layers are visible. The smallest non-repeating section of the Patterson is shown (a). The main features can be identified with interatomic vectors in a dimer model of the surface (b), slight distortion in the Patterson (the vectors do not run through the middle of the rings) being due to the absence of the integer-order reflections. The size of the unit cell doubles in one direction when the top layer atoms dimerize (c).

(a)

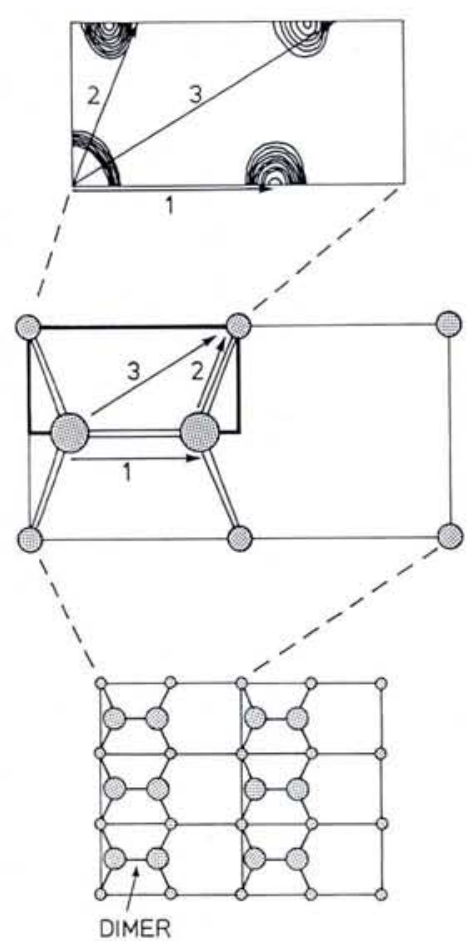

problems is to use a miniature ultra-high vacuum chamber which can be disconnected from a larger sample preparation chamber and mounted on a diffractometer. Recently, though, several instruments have been built where the sample can be prepared and studied with X-ray diffraction in the same chamber, which permits a much wider range of experiments to be performed [2].

The steps from the surface X-ray diffraction measurements to the analysis of the data are straightforward: structure factor intensities are obtained from the integrated, background-subtracted intensities of the Bragg reflections after correction for the polarization factor and certain geometric factors connected with the scattering geometry. It must be borne in mind, though, that the data provides only the amplitude of the structure factors, and not their phases. Since the beginnings of $\mathrm{X}$-ray crystallography, a great variety of ingenious methods have been devised to extract structural information from diffraction data, and thus side-step the famous "phase-problem". One of the strengths of the surface X-ray diffraction technique is that it can immediately draw on this bank of knowledge.

\section{In-plane Structure}

The first step in the data analysis is to plot the Fourier transform of the X-ray intensities, familiarly called the Patterson function. Because this is a transform of structure factor intensities, it does not yield the electron density in the unit cell, but rather the autocorrelation function of that density. The Patterson function will have peaks at positions relative to the origin which correspond to interatomic distances in the unit cell, since the autocorrelation integral will have a maximum when two atoms overlap. Such a function is plotted in Fig. 2a, based on data from a surface X-ray diffraction measurement of the $\mathrm{Ge}(001) 2 \times 1$ reconstructed surface [3].

In common with most surface X-ray structure determinations to date, the measurements were made with grazing angles of incidence and exit, so that only a plane in reciprocal space was probed. As a consequence, the Patterson function is that of the $2 \times 1$ unit cell projected onto the surface plane. Another important point is that reflections with integer Miller indices are not used in the analysis, because there is a coherent contribution to these reflections from the substrate. This systematic absence of structure factor intensities tends to distort the Patterson function slightly. Despite these shortcomings, three interatomic distances are clearly visible in Fig. 2a. It is possible to eliminate several of the many structural models that have been proposed for this surface, simply by comparison with the Patterson function. Fig. $2 b$ shows the projected structure of a model in which the surface form dimers in order to saturate the broken bonds at the surface. The figure indicates the three interatomic vectors which are significantly distorted from the ideal surface geometry, corresponding to the features in the Patterson.

Once a model has been found which can account for the Patterson, a more

\section{EUROPEAN SYNCHROTRON RADIATION FACILITY \\ GRENOBLE - FRANCE}

The ESRF, is an international synchrotron radiation source to be built in Grenoble, France. It will consist of a $6 \mathrm{GeV}$ electon/positron storage ring surrounded by $\mathrm{X}$-ray beam-lines. Our Experiments Division is looking for a

\section{SCIENTIST}

\section{TO TAKE CHARGE OF HIGH X-RAY ENERGY SCATTERING}

Basic experience required: An international reputation for research involving high energy (> $50 \mathrm{keV}) \mathrm{X}$-rays.

Duties: To be responsible for the design and construction of beam-lines for high energy $X$-ray scattering and to provide scientific leadership in this area at ESRF. One of the characteristic properties of ESRF is that it will produce $X$-ray beams of high energy and low emittance. The scientist in charge of high energy $\mathrm{X}$-ray scattering will be required to develop a relationship with users from countries participating in ESRF. $\mathrm{He} / \mathrm{She}$ would liaise with the Optics Group and the Detector Group at ESRF in research and development projects on the problems of constructing beam lines to match the quality of the planned ESRF beams. He/She should speak fluent English.

Remuneration: Gross annual salary from 250 to $300 \mathrm{kFF}$ depending on qualifications and experience. In addition: monthly family supplement and expatriation allowance for non-French staff (calculated on the basis of family situation) and a settling in allowance and adaptation allowance (paid once).

Applications should be sent, in English or French, with the names of three referees before 30 SEPT. 88 , to:

ESRF-Personnel Office - Ref. 17/2111 - BP 220 - F 38043 GRENOBLE CEDEX 

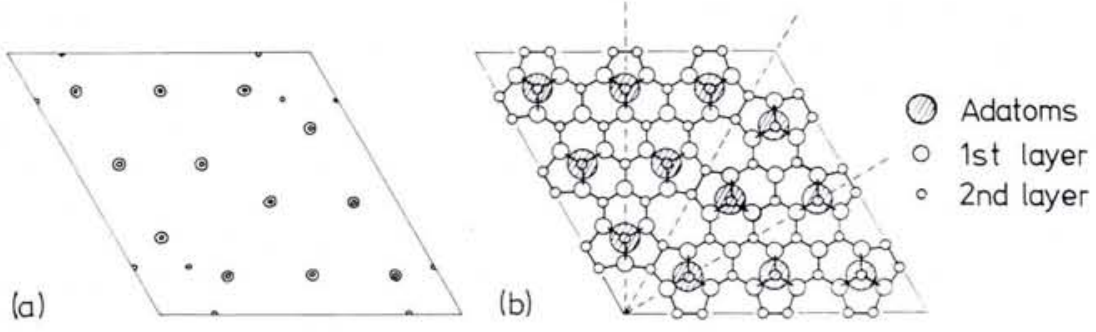

Fig. 3-Electron density difference map of $\mathrm{Sn} / \mathrm{Ge}(111) 7 \times 7$. The structure is closely related to that of the Dimer-Adatom-Stacking fault (DAS) model of the Si(111)7 $\times 7$ surface. A map of electron density difference (a) is given by

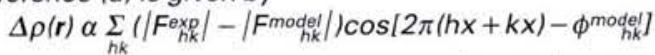

where $/ F^{e x p}$ hk $/$ are the experimental structure factors and $/ F^{\text {model }}{ }_{h k} /$ are the structure factors for a DAS model containing only Ge atoms. The phases of the experimental structure factors are unknown and therefore assumed to be $\Phi_{\text {model }}{ }_{h k}$, the phases for the model structure factors. The result shows missing electron density on the adatom positions (shaded in b). The adatoms are thus identified to be $\mathrm{Sn}$.

detailed fit of the structural parameters can be performed, in which the atom positions are varied to minimize a leastsquares residual between the experimental and calculated structure factor intensities. This fitting procedure is straightforward, but there is no guaranty of success. For simple unit cells, such as that of $\mathrm{Ge}(001)$, satisfactory agreement is obtained with the model suggested by the Patterson function. But for structures with large unit cells it may be necessary to include extra atoms that are not immediately apparent from the Patterson function. These extra atoms will appear in an electron density difference map. This is obtained by subtracting the measured structure factor amplitudes from those of the best (but inadequate) model, assuming the structure factor phases of the model, and performing a Fourier inversion. If the starting model is not too different from the actual structure, then this procedure will reveal where there is too much or too little electron density in the model unit cell.

An elegant example of the use of electron density difference maps comes from a study of a $7 \times 7$ reconstruction on the $\mathrm{Ge}(111)$ surface, induced by the adsorption of a submonolayer amount of $\mathrm{Sn}$. This study involved the collection of 269 different reflections, the largest surface X-ray diffraction data set to date. The structure was found to be identical to the generally accepted DimerAdatom-Stacking fault (DAS) model for the $\mathrm{Si}(111) 7 \times 7$ surface. Since $\mathrm{Sn}$ has more electrons than $\mathrm{Ge}$, it is possible to find which sites the Sn atoms occupy by performing an electron difference map between the data and a DAS model containing only $\mathrm{Ge}$ atoms. The result is shown in Fig. 3a. The peaks in the map are the positions of the adatoms in the unit cell (the shaded atoms in Fig. 3b), indicating that $\mathrm{Sn}$ substitutes $\mathrm{Ge}$ at (a)

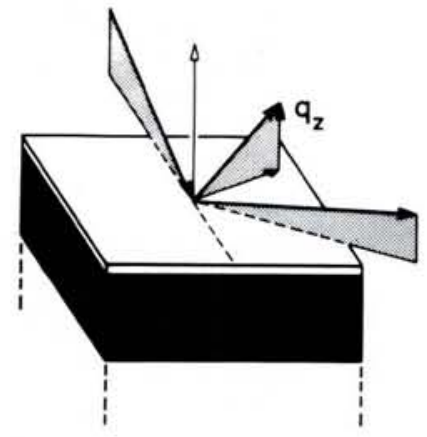

(b)

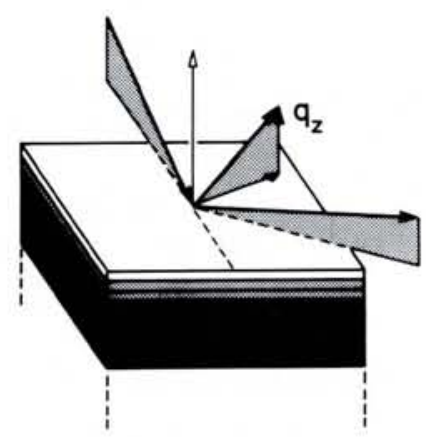

(c)

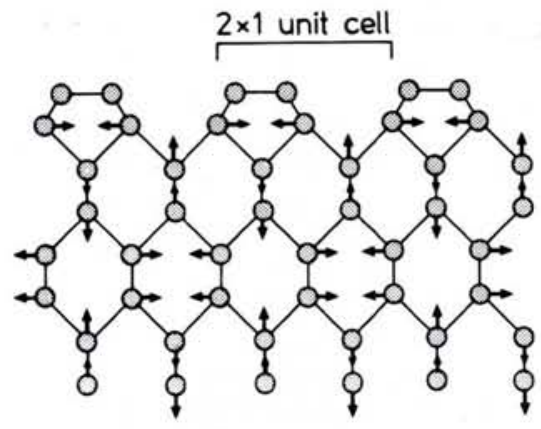

these sites [4]. The same pattern of twelve dots is familiar to surface scientists from the scanning-tunnelling $\mathrm{mi}$ croscope pictures of the $\mathrm{Si}(111) 7 \times 7$ unit cell obtained by Binnig and Rohrer.

The methods used for the determination of the projected structure of the unit cell belong to the standard analysis techniques of X-ray diffraction. But there are two important features that distinguish surface $\mathrm{X}$-ray diffraction from X-ray diffraction experiments on bulk crystals: the two-dimensional nature of the surface, and the interference of surface and bulk scattering. Both features offer the possibility of obtaining additional information about the surface structure.

Fig. 4 - Schematic illustration of scattering along a Bragg rod of intensity from a reconstructed surface. If only one layer is different from the ideal bulk geometry, there is monotonic decay of intensity along the rod (a). If several subsurface layers are perturbed by the reconstruction, they contribute to the Bragg rod too: the intensity has an oscillatory variation due to interference of the scattering from different layers (b). For Ge(001), the Bragg rods have pronounced oscillatory behaviour, which can be accounted for by elastic deformation of the subsurface layers due to the dimers at the surface. The data is well-fitted by a model in which the first eight layers are relaxed (full line in c).
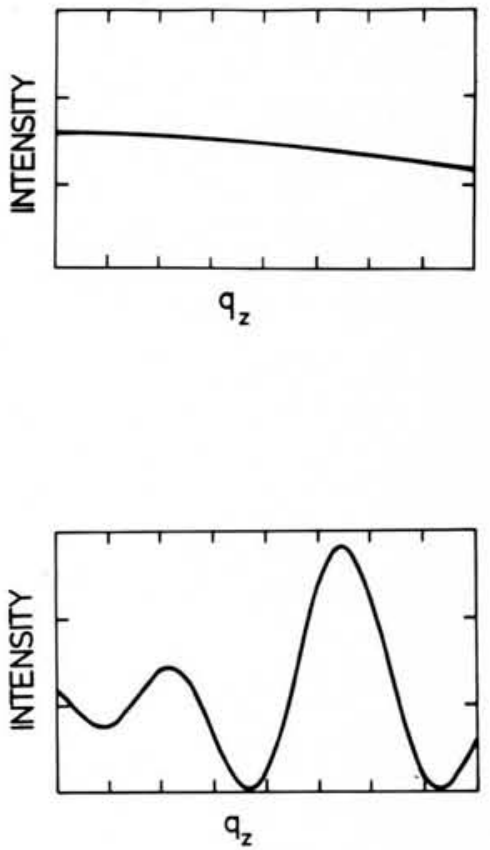

Ge (001) $2 \times 1$

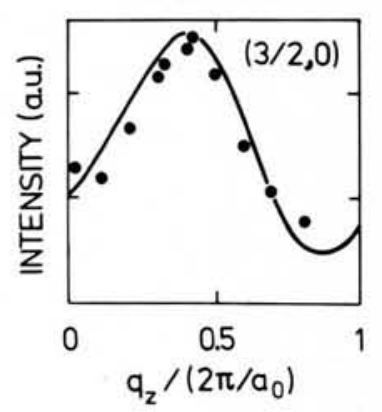


Bragg Rods and Subsurface Relaxation

The two-dimensional periodicity of a reconstructed surface translates into an array of rods in reciprocal space, extending perpendicular to the surface plane. The reciprocal lattice rods are defined by three Miller indices, $h$ and $k$ in the plane of the surface, and by a continuous index I along the rod. There are no restrictions on the third index simply because the reconstructed unit cell does not repeat perpendicular to the surface.

If only a single atomic layer were involved in the reconstruction, then the rods would be essentially featureless, except for the monotonic dependence on the atomic form-factor and thermal Debye-Waller factor. In practice, the reconstruction perturbs several subsurface layers. These layers are distorted slightly from their bulk positions, acquiring the same periodicity as the surface. They therefore contribute to the fractional-order Bragg reflections. This contribution is particularly apparent along the Bragg rods, which become modulated (see Fig. 4).

An example of a modulated Bragg rod for the $\mathrm{Ge}(001)$ surface is given in Fig. 4. To account for the experimental modulation it is necessary to include relaxations in the first eight layers of the crystal. The relaxations of the subsurface layers are of the order of $0.1 \AA$, or less than $5 \%$ of the bulk $\mathrm{Ge}$ bond-length. The subsurface relaxation occurs in order to minimize the strain induced by dimerization of the top $\mathrm{Ge}$ layer. The relaxation patterns for this and other reconstructed semiconductor surfaces can be understood from the elastic properties of $\mathrm{Si}$ and $\mathrm{Ge}$ : bond stretching is energetically more expensive than bond bending, causing the atoms to relax so as to preserve bond-length as far as possible.

\section{Crystal Truncation Rods and Adsorbate Registry}

The other unique feature of surface $\mathrm{X}$-ray diffraction is the coherent combination of scattering from the substrate and from the surface reconstruction for Bragg reflections with integer Miller indices $h, k$. The sharp interface between crystal and vacuum leads to tails of intensity about Bragg reflections, extending along the direction normal to the surface (see Fig. 5). Between reflections, the intensity in these "crystal truncation rods" is comparable to that due to a single atomic layer, and thus easily measurable in a surface $X$-ray diffraction experiment [5].

For a reconstructed surface, the crystal can conveniently be divided into two regions, a near-surface region of one or several layers for which the deviations from the ideal bulk structure are large, and deeper layers which are essentially unperturbed. The scattering at the integer-order reflections is then the coherent combination of a Bragg rod from the surface region, and the crystal truncation rod of the substrate. The sum of these two will depend sensitively on the position of adsorbate atoms in the unit cell, and measurements of the integer-order reflections can thus be used to determine the registry of a mesh formed by atoms adsorbed on the crystal, and thus deduce the preferred adsorption sites. This technique has been applied to the $\sqrt{3} \times \sqrt{3} \mathrm{R} 30$ reconstructions of $\mathrm{Pb}$ chemisorbed on the $\mathrm{Ge}$ (111) surface [6]. A simple structure, called the $\alpha$-phase, occurs for a coverage of 1 $\mathrm{Pb}$ atom per unit cell. In the denser $\beta$ phase, the sites between the $\mathrm{Pb}$ atoms at the corners of the unit cell are filled so that there are $4 \mathrm{~Pb}$ atoms per unit cell, resulting in a dense-packed $\mathrm{Pb}$ layer (Fig. 6). Although these structural models for the two phases had been proposed over twenty years ago on the basis of electron diffraction measurements, the $\mathrm{Pb}$ adsorption sites were not known. For the simple $\alpha$-phase, analysis of the integer-order reflections showed that the $\mathrm{Pb}$ atoms sit in triangles of top layer $\mathrm{Ge}$ atoms and above second layer atoms. In the $\beta$-phase, though, the analysis of the integer order reflections

\title{
PLASMA PHYSICS AND CONTROLLEDFUSION
}

\author{
AEurophysics JournalPublished jointly by theInstitute of Physicsand Pergamon Press \\ Honorary Editor: A GIBSON, JET Joint Undertaking, Abingdon, Oxfordshire OX143EA, UK
}

A monthly journal for the communication of the results of current research on all aspects of plasma physics and controlled fusion. It is one of the leading international journals in this field. Research papers published cover all aspects of plasma physics and controlled nuclear fusion together with the plasma physics of highly ionised gases, high temperature, collective processes, and other fusion oriented research.

\section{A Selection of Papers}

Review of Tokamak plasma heating by wave damping in the ion cyclotron range of frequency, J ADAM (France).

Langmuir probe measurements in the TEXTOR Tokamak during ALT-I pump limiter experiments, D M GOEBEL (USA) et al.

Characteristics of Alfven surface waves along moving cylindrical plasma columns, K SOMASUNDARAM (India) \&

A SATYA NARAYANAN (India).

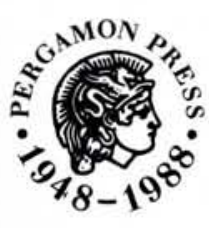

PERGAMONPRESSInc

USA, Central and South America:

FairviewPark,Elmsford,

New York 10523 , USA
On the quasilinear renormalization theory of turbulent magnetized plasma, C F ZHANG (China).

A method for time resolved neutron spectroscopy on short pulsed fusion neutron sources, R SCHMIDT (FRG) \& H HEROLD (FRG)

Convectiveequilibrium and mixing-length theory for stellarator reactors, D D M HO (USA) \& R M KULSRUD (USA).

Indexed/Abstracted in: Current Contents, Chemical Abstracts, PASCAL-CNRS Database, INSPEC

Subscription Information

1988: Volume 30 (14 issues) Annual rate (1988): DM 990.00 German Mark (DM) prices quoted applyin Europe, Africa, Asia and Australia (with theexceptionoflapan). For therestof the world apply to thenearest Pergamonoffice.

Pergamon accepts UNESCO coupons. Prices are subject to change without notice. Prices include postage and insurance.

Back issues of all volumes of Pergamon journals are available in hard copy from Pergamon Press. Please send for a separate price list. Subscribers ordering a current subscription may order back issues at a $25 \%$ discount.

PERGAMONPRESS plc

UKandallothercountries:

HeadingtonHillHall,

OxfordOX30BW, UK

FREE SAMPLE COPIES AVAILABLE ON REQUEST 
clearly indicates a shift of the unit cell so that the corner atoms sit on a new adsorption site, above 4 th layer $\mathrm{Ge}$ atoms (Fig. 6). The $\beta$-phase is not, therefore, produced by simply "filling the gaps" in the $\alpha$-phase, but by a significant chemical change at the surface, involving a new bond configuration.

The few examples given here illustrate the potential of X-ray diffraction for the investigation of surface crystallography.
The technique is unique owing to both the simplicity of the analysis, and because X-rays can probe the structure of layers below the surface. Combining the in-plane structure with the information extracted from the reciprocal lattice rods gives surface $\mathrm{X}$-ray diffraction a very wide scope: it should in future become a routine matter to determine the full three-dimensional structure of a reconstructed surface.

Fig. 5 - The principle of crystal truncation rods. The intensity along a Bragg rod passing through bulk Bragg reflections is shown. For a slab of N layers the scattered intensity has the form: $I=I_{0}\left[\sin ^{2}\left(N q_{z} a_{o} / 2\right)\right] /\left[\sin ^{2}\left(q_{z} a_{o} / 2\right)\right]$ where $I_{o}$ is the scattered intensity from a single layer (a). The result is analogous to the multiple-slit diffraction pattern in optics. If the crystal is allowed to extend to infinity in one direction, the resulting scattered intensity has the form: $I=I_{\sigma} / \sin ^{2}\left(q_{z} a_{o} / 2\right)$ which is the envelope-function of the scattering from a finite crystal (b). Between the Bragg reflections, the intensity in the crystal truncation rod falls to $I_{o}$, the scattered intensity from a single layer.
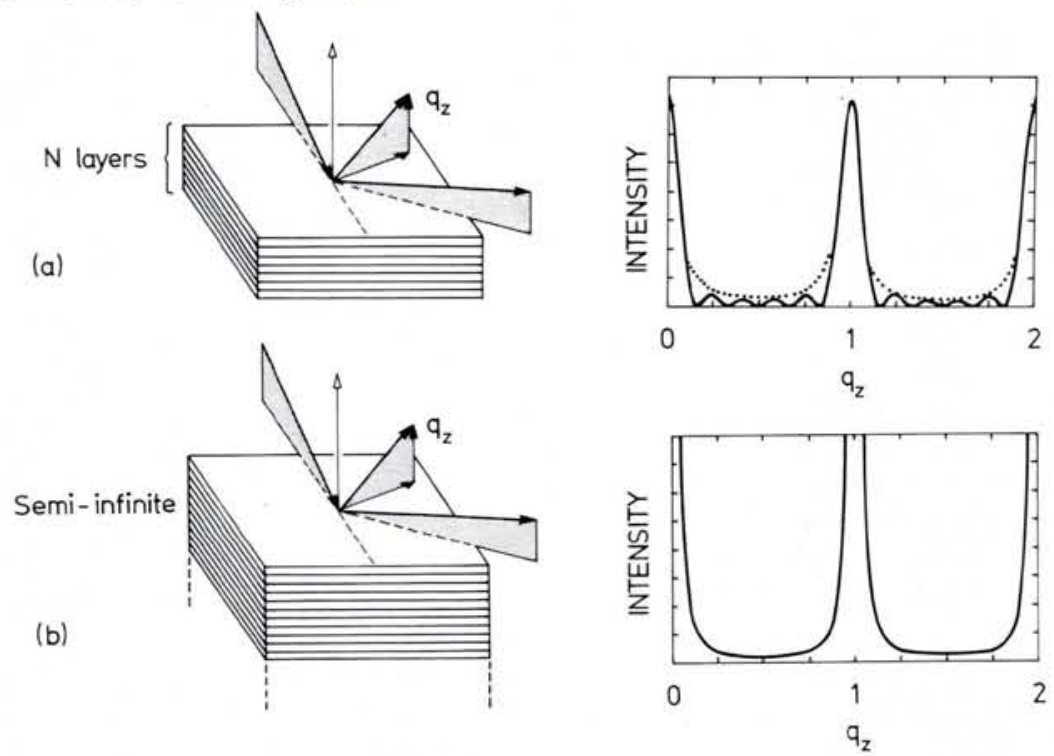

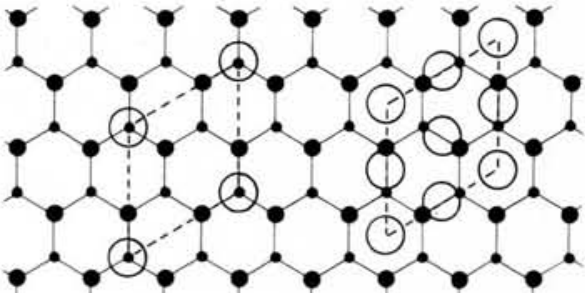

$$
\alpha \text {-phase } \quad \beta \text {-phase }
$$

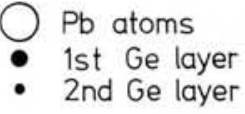

Fig. 6 - The $\alpha$ and $\beta$ phases of $\mathrm{Pb} / \mathrm{Ge}(111)$ $\sqrt{ } 3 \times \sqrt{ } 3$. The dilute $\alpha$-phase has one atom per unit cell situated above the second layer $\mathrm{Ge}$ atom. In the $\beta$ phase, $\mathrm{Pb}$ atoms form a dense-packed structure. From the analysis of the in-plane integer-order reflections it can be deduced that the corner atoms of the unit cell are shifted to a new adsorption site in the $\beta$ phase, in the holes of the honeycomb pattern formed by the first Ge bilayer (directly above the fourth layer $\mathrm{Ge}$ atoms). Note that the other atoms in the $\beta$ phase are not on high-symmetry sites.

\section{REFERENCES}

[1] Eisenberg P. and Marra W.C., Phys. Rev. Lett. 46 (1981) 1081.

[2] Robinson I., in Handbook of Synchrotron Radiation, Eds. D.E. Moncton and G. Brown (North Holland) 1987.

[3] Grey F. et al., The Structure of Surfaces II, Eds. J.F. van der Veen and M.A. van Hove (Springer Verlag) 1988, p. 292.

[4] Pedersen J. Skov et al., ibid., p. 352.

[5] Robinson I., Phys. Rev. B 33 (1986) 3830.

[6] Feidenhans'। R. et al., Surf. Sci. 178 (1986) 927.

\section{New Members of the European Physical Society}

\section{CATEGORY 4a)}

E. Maniataki, Heraklio, Crete, GR L.V. Woodcock, Bradford, UK

\section{CATEGORY 4c)}

Belgian Physical Society

J. Delhalle, Namur

W. Malfliet, Wilrijk

M. Terao, Belfast, UK

R.R. Weynants, Leuven

\section{Danish Physical Society}

B. Kweri, Rødovre

A. Luther, Copenhagen

S. Steenstrup, Copenhagen

Eötvös Lorand Physical Society

P. Vanko, Hamburg

Finnish Physical Society

E.K. Suokko, Kouvola

German Physical Society

H.-P. Eckle, Berlin

K.-H. Rieder, Berlin
French Physical Society

S. Aubry, Palaiseau

Y. Lapierre, Gif-sur-Yvette

G. Lelay, Marseille

J. Prost, Paris

Hellenic Physical Society

E. Syskakis, Nofalia-Neapolis, Crete

The Institute of Physics

R.J.P. Bain, Glasgow

Italian Physical Society

S. Atzeni, Frascati

A. Giulietti, Pisa

G. Godoli, Firenze

G. Lampis, Milan

A. Waheed, Legnaro

The Netherlands' Physical Society

J. Bergsma, Bergen

C.T.A.M. de Laat, Utrecht

F.H.M. Spit, Utrecht

T.P. Valckering, Oldenzaal
Union of Czechoslovak

Mathematicians and Physicists

M. Demjanenko, Prague

Polish Physical Society

R. Kutner, Warsaw

Z.A. Lalak, Warsaw

K.J. Zablocki, Warsaw

Portuguese Physical Society M.J. Martins, Lisbon

Spanish Royal Society of Physics

N. Barberan, Barcelona

A. Fernandez-Ranada, Madrid

A. Pacheco Pagés, Bellaterra

F. Soria-Gallego, Madrid

Swedish Physical Society C.G. Fälthammar, Stockholm

B. Gustafsson, Uppsala

S. Mannervik, Stockholm

G. Ohlén, Lund
Swiss Physical Society

T.-M. Tran, Lausanne

\section{CATEGORY 4d)}

American Physical Society

L.S. Cutler, Los Altos, CA

L. Irakliotis, Ioannina, GR

K.K. Phua, Singapore

A. Pumir, Gif-sur-Yvette, F

S.M. Raza, Quetta, Pakistan

M.C. Ross, Stanford, CA

French Optical Society

C. Cahen, Chatou

Y. May, Vélizy

J.-C. Perrin, Le Plessis Robinson

J. Taboury, Orsay

S. Vatoux, Orsay

Physical Society of Japan

Y. Hiwatari, Kanazawa

M. Izumi, Tokyo 